\title{
Bayesian Analysis of the Behrens-Fisher Problem under a Gamma Prior
}

\author{
Nengak Emmanuel Goltong*, Sani Ibrahim Doguwa \\ Department of Statistics, Ahmadu Bello University, Zaria, Nigeria \\ Email: *spomariae@gmail.com
}

How to cite this paper: Goltong, N.E. and Doguwa, S.I. (2018) Bayesian Analysis of the Behrens-Fisher Problem under a Gamma Prior. Open Journal of Statistics, 8 , 902-914.

https://doi.org/10.4236/ojs.2018.86060

Received: October 27, 2018

Accepted: December 1, 2018

Published: December 4, 2018

Copyright $\odot 2018$ by authors and Scientific Research Publishing Inc. This work is licensed under the Creative Commons Attribution International License (CC BY 4.0).

http://creativecommons.org/licenses/by/4.0/

\begin{abstract}
Yin [1] has developed a new Bayesian measure of evidence for testing a point null hypothesis which agrees with the frequentist p-value thereby, solving Lindley's paradox. Yin and Li [2] extended the methodology of Yin [1] to the case of the Behrens-Fisher problem by assigning Jeffreys' independent prior to the nuisance parameters. In this paper, we were able to show both analytically and through the results from simulation studies that the methodology of Yin [1] solves simultaneously, the Behrens-Fisher problem and Lindley's paradox when a Gamma prior is assigned to the nuisance parameters.
\end{abstract}

\section{Keywords}

Behrens-Fisher Problem, Lindley's Paradox, Metropolis-Hastings Algorithm, Informative Priors

\section{Introduction}

Consider a hypothesis testing problem about the difference of two means as follows: Let $X_{1} \sim N\left(\mu_{1}, \sigma_{1}^{2}\right)$ and $X_{2} \sim N\left(\mu_{2}, \sigma_{2}^{2}\right)$ and no assumption is made about $\sigma_{1}^{2}$ and $\sigma_{2}^{2}$. Then testing the hypothesis stated as

$$
H_{0}: \theta=\mu_{1}-\mu_{2}=0 \text { Versus } H_{0}: \theta \neq 0
$$

based on random samples of size $n_{1}$ and $n_{2}$ respectively, and the assumption that $X_{1}=\left(X_{11}, X_{12}, \cdots, X_{1 n_{1}}\right)$ and $X_{2}=\left(X_{21}, X_{22}, \cdots, X_{2 n_{2}}\right)$ are independent is known as the Behrens-Fisher problem.

Lindley [3] showed that using the same data, the conclusion of a hypothesis test from a frequentist perspective could differ from that of a Bayesian perspective. It was shown that as $n \rightarrow \infty$, the posterior probability under $H_{0}$ tends to 1 . This result holds irrespective of the prior probability assigned to $H_{0}$. For discussions and arguments concerning Lindley's paradox, see Spanos [4] and Robert 
[5]. As an extension of the methodology of Yin [1], Yin and Li [2] made an attempt to solve the Behrens-Fisher Problem as well as Lindley's Paradox using a noninformative prior. In this work, we propose to examine the performance of the methodology of Yin [1] in solving simultaneously, the Behrens-Fisher problem and Lindley's paradox when Gamma priors are assigned to the unknown variances.

\section{Literature Review}

Scheffe [6] showed that for the Behrens-Fisher problem, there does not exist convenient tests and confidence intervals by constructing a test statistic based on a linear and a quadratic form. He established this result by showing that there exists no symmetric solution to the Behrens-Fisher problem using this approach. Fraser and Streit [7] derived a valid solution for the Behrens-Fisher problem using arbitrary absolutely continuous error distributions. They used a structural approach, where the random fluctuations apparent in the experiment were generated by a random variable with known distribution. Robinson [8] investigated the discrepancy between the coverage probabilities for the Behrens-Fisher intervals and the intervals of the nominal significance level. He advocates the use of the Behrens-Fisher test unless a proper Bayesian test is considered appropriate. Tsui and Weerahandi [9] proposed the use of generalized pivotal quantities and generalized p-values in the case of hypothesis testing in the presence of nuisance parameters given by

$$
p(x)=\operatorname{Pr}\left[F_{1, n_{1}+n_{2}-2} \geq\left(\bar{x}_{1}-\bar{x}_{2}\right)^{2}\left(n_{1}+n_{2}-2\right)\left(\frac{\tilde{s}_{1}^{2}}{B}+\frac{\tilde{s}_{2}^{2}}{1-B}\right)^{-1}\right]
$$

where $\tilde{s}_{i}^{2}=\left(1-n_{i}^{-1}\right) s_{i}^{2}, \quad i=1,2, s_{i}^{2}$ is the variance of sample $i$ calculated from a sample of size $n_{i}$ and $B \sim \operatorname{Beta}\left(\frac{\left(n_{1}-1\right)}{2}, \frac{\left(n_{2}-1\right)}{2}\right)$. Zheng et al. [10] proposed an approach to solving the Behrens-Fisher problem in such a way that the Type-II error and the length of the confidence interval is controlled conditioned on a specified Type-I error by using Stein's two-stage sampling scheme. Ozkip et al. [11] compared the different methods of solving the Behrens-Fisher problem to see which test outperforms the rest.

Degroot [12] commented on the reaction of some Bayesians on the use of informative priors. He disagrees with the notion that diffuseness of a prior distribution reflects ignorance about the distribution of such a parameter. Berger and Sellke [13] investigated the relationship between the p-value and the Bayesian measure of evidence against the null hypothesis for a two-sided hypothesis testing problem and concluded that the two measures of evidence were irreconcilable. Casella and Berger [14] investigated the discrepancy between the Bayesian measure of evidence, that is, the posterior probability that $H_{0}$ is true, and the p-value, in a one-sided hypothesis testing problem under the same class of priors as in Berger and Sellke [13] but concluded that the two measures of evidence 
were reconcilable. Berger and Delampady [15] also investigated the discrepancy between the p-value and the Bayesian measure of evidence and concluded that using a noninformative prior does not necessarily solve Lindley's paradox.

Meng [16] proposed a Bayesian counterpart of the generalized p-value to allow the "Test Statistic" depend on both the data and unknown (nuisance) parameters and thus permit a direct measure of the discrepancy between sample and population quantiles. Unlike the generalized p-value of Tsui and Weerahandi [9] that requires the use of a pivotal quantity as a test variable whose tail area probabilities are free of nuisance parameters, only the specification of prior distributions are required for the posterior predictive $\mathrm{p}$-value which is given as

$$
\operatorname{ppp}(x)=\operatorname{Pr}\left\{F_{1, n_{1}+n_{2}} \geq \frac{\left(\bar{x}_{1}-\bar{x}_{2}\right)^{2}\left(n_{1}+n_{2}\right)}{\left[\tilde{s}_{1}^{2}+\left(\bar{x}_{1}-\mu\right)^{2}\right] B_{n_{1}, n_{2}}^{-1}+\left[\tilde{s}_{2}^{2}+\left(\bar{x}_{2}-\mu\right)^{2}\right]\left(1-B_{n_{1}, n_{2}}\right)^{-1}}\right\}
$$

where $\tilde{s}_{i}^{2}=\left(1-n_{i}^{-1}\right) s_{i}^{2}, i=1,2$ and $B_{n_{1}, n_{2}} \sim \operatorname{Beta}\left(\frac{n_{1}}{2}, \frac{n_{2}}{2}\right)$.

Ghosh and Kim [17] proposed an approach of constructing a prior different from Jeffreys' independent prior that leads to a credible interval whose asymptotic coverage probability matches the frequentist coverage probability more accurately than Jeffreys' interval. Yin [1] developed a Bayesian testing procedure that solves Lindley's Paradox in testing a precise null hypothesis in the one sample case. Instead of the conventional Bayesian approach, this new procedure avoids the dichotomy of the parameter space. Let $X_{1}, X_{2}, \cdots, X_{n}$ be a random sample from a distribution with density $f(x \mid \theta)$, where $\theta$ is unknown and belongs to the parameter space $\Theta$, and let $\theta$ have a prior density $\pi(\theta)$, then the new Bayesian measure of evidence is

$$
P^{B}(x)=P\left(|\theta-E(\theta \mid x)| \geq\left|\theta_{0}-E(\theta \mid x)\right| \mid x\right)
$$

where $E(\theta \mid x)$ is the posterior expectation of $\theta$ under the prior $\pi(\theta)$. A smaller value of $P^{B}(x)$ means a bigger distance between $\theta_{0}$ and the true $\theta$ and therefore, suggests stronger evidence against the null hypothesis $H_{0}$.

Yin and Li [2] extended the methodology of Yin [1] to solve simultaneously, the Behrens-Fisher problem and Lindley's Paradox using Jeffreys' objective prior given as

$$
\pi\left(\mu_{1}, \mu_{2}, \sigma_{1}^{2}, \sigma_{2}^{2}\right) \propto \frac{1}{\sigma_{1}^{2} \sigma_{2}^{2}}
$$

They showed that the posterior distribution of $\theta$, the difference of the two means $\mu_{1}$ and $\mu_{2}$ is given as

$$
\theta \mid x \sim \bar{x}_{1}-\bar{x}_{2}-\left(\frac{S_{1} T_{n_{1}-1}}{\sqrt{n_{1}}}-\frac{S_{2} T_{n_{2}-1}}{\sqrt{n_{2}}}\right)
$$

and the Bayesian measure of evidence under Jeffreys' independent prior was given by 


$$
P_{J}^{B F}(x)=P\left(\left|\frac{S_{1} T_{n_{1}-1}}{\sqrt{n_{1}}}-\frac{S_{1} T_{n_{1}-1}}{\sqrt{n_{1}}}\right| \geq\left|\bar{x}_{1}-\bar{x}_{2}\right|\right)
$$

where $T_{a-1}$ is a $t$ variable with $a-1$ degrees of freedom. This approach was shown to solve the two problems and also, to yield credible intervals that actually possess $1-\alpha$ coverage probability. This was however only demonstrated where a non-informative prior was used and the use of an informative prior was recommended in order to see how the methodology performs under such conditions.

\section{Main Results}

Let there exist samples of sizes $n_{1}$ and $n_{2}$ from $X_{1} \sim N\left(\mu_{1}, \sigma_{1}^{2}\right)$ and $X_{2} \sim N\left(\mu_{2}, \sigma_{2}^{2}\right)$ respectively. Under the assumption of independence, and letting $X=\left(x_{1}, x_{2}\right)$, the likelihood function is given by

$$
f\left(x \mid \mu_{1}, \mu_{2}, \sigma_{1}^{2}, \sigma_{2}^{2}\right) \propto \sigma_{1}^{-n_{1}} \sigma_{2}^{-n_{2}} \exp \left[-\frac{1}{2} \sum_{i=1}^{2} \frac{S_{i}\left(\mu_{i}\right)}{\sigma_{i}^{2}}\right]
$$

where

$$
S_{i}\left(\mu_{i}\right)=\sum_{j=1}^{n_{i}}\left(x_{i j}-\mu_{i}\right)^{2}
$$

Now, let $\pi\left(\mu_{1}, \mu_{2}, \sigma_{1}^{2}, \sigma_{2}^{2}\right) \propto 1, \tau_{i}=\sigma_{i}^{-2}$, and $\pi\left(\tau_{i}\right) \sim \operatorname{Gamma}\left(\alpha_{i}, \beta_{i}\right), i=1,2$. The marginal posterior distribution of $\left(\mu_{1}, \mu_{2} \mid x\right)$ is given by

$$
\begin{aligned}
& \pi^{G a}\left(\mu_{1}, \mu_{2} \mid x\right) \\
& \propto \iint_{0}^{\infty} \pi^{G a}\left(\mu_{1}, \mu_{2}, \sigma_{1}^{2}, \sigma_{2}^{2} \mid x\right) \mathrm{d} \sigma_{1}^{2} \mathrm{~d} \sigma_{2}^{2} \\
& \propto \iint_{0}^{\infty} \sigma_{1}^{-n_{1}} \sigma_{2}^{-n_{2}} \exp \left[-\frac{1}{2} \sum_{i=1}^{2} \frac{S_{i}\left(\mu_{i}\right)}{\sigma_{i}^{2}}\right] \tau_{1}^{\alpha_{1}-1} \tau_{2}^{\alpha_{2}-1} \mathrm{e}^{-\left(\tau_{1} \beta_{1}+\tau_{2} \beta_{2}\right)} \mathrm{d} \sigma_{1}^{2} \mathrm{~d} \sigma_{2}^{2}
\end{aligned}
$$

So, we have that

$$
\begin{aligned}
& \pi^{G a}\left(\mu_{1}, \mu_{2} \mid x\right) \\
& \propto\left[\frac{1}{2} S_{1}\left(\mu_{1}\right)+\beta_{1}\right]^{-\left(n_{1}+2 \alpha_{1}-4\right) / 2}\left[\frac{1}{2} S_{2}\left(\mu_{2}\right)+\beta_{2}\right]^{-\left(n_{2}+2 \alpha_{2}-4\right) / 2} \\
& \propto\left[1+\frac{n_{1}\left(\bar{x}_{1}-\mu_{1}\right)^{2}+2 \beta_{1}}{\left(n_{1}-1\right) S_{1}^{2}}\right]^{-\left(n_{1}+2 \alpha_{1}-4\right) / 2}\left[1+\frac{n_{2}\left(\bar{x}_{2}-\mu_{2}\right)^{2}+2 \beta_{2}}{\left(n_{2}-1\right) S_{2}^{2}}\right]^{-\left(n_{2}+2 \alpha_{2}-4\right) / 2}
\end{aligned}
$$

Let

$$
a_{i}=1+\frac{2 \beta_{i}}{\left(n_{i}-1\right) S_{i}^{2}}, i=1,2
$$

Then we have that

$$
\pi^{G a}\left(\mu_{1}, \mu_{2} \mid x\right) \propto\left[1+\frac{n_{1}\left(\bar{x}_{1}-\mu_{1}\right)^{2}}{a_{1}\left(n_{1}-1\right) S_{1}^{2}}\right]^{-\left(n_{1}+2 \alpha_{1}-4\right) / 2}\left[1+\frac{n_{2}\left(\bar{x}_{2}-\mu_{2}\right)^{2}}{a_{2}\left(n_{2}-1\right) S_{2}^{2}}\right]^{-\left(n_{2}+2 \alpha_{2}-4\right) / 2}
$$


Now, let

$$
t_{i}=\frac{\sqrt{n_{i}\left(n_{i}+2 \alpha_{i}-5\right)}\left(\bar{x}_{i}-\mu_{1}\right)}{\sqrt{a_{i}\left(n_{i}-1\right)} S_{i}}
$$

And then (11) simplifies to

$$
\pi^{G a}\left(\mu_{1}, \mu_{2} \mid x\right) \propto\left[1+\frac{t_{1}^{2}}{n_{1}+2 \alpha_{1}-5}\right]^{-\left(n_{1}+2 \alpha_{1}-4\right) / 2}\left[1+\frac{t_{2}^{2}}{n_{2}+2 \alpha_{2}-5}\right]^{-\left(n_{2}+2 \alpha_{2}-4\right) / 2}
$$

where clearly, (12) is the kernel of the joint distribution of two independent $t$ random variables $t_{1}$ and $t_{2}$ with $n_{1}+2 \alpha_{1}-5$ and $n_{2}+2 \alpha_{2}-5$ degrees of freedom respectively.

Also, we have that

$$
\begin{aligned}
\mu_{i} & =\bar{x}_{i}-\frac{t_{i} S_{i} \sqrt{a_{i}\left(n_{i}-1\right)}}{\sqrt{n_{i}\left(n_{i}+2 \alpha_{i}-5\right)}} \\
& =\bar{x}_{i}-\frac{t_{i} \sqrt{S_{i}^{2}\left(n_{i}-1\right)\left(1+\frac{2 \beta_{i}}{\left(n_{i}-1\right) S_{i}^{2}}\right)}}{\sqrt{n_{i}\left(n_{i}+2 \alpha_{i}-5\right)}} \\
& =\bar{x}_{i}-\frac{t_{i} \sqrt{\left(n_{i}-1\right) S_{i}^{2}+2 \beta_{i}}}{\sqrt{n_{i}\left(n_{i}+2 \alpha_{i}-5\right)}}
\end{aligned}
$$

And this implies that the posterior distribution of $\theta$, the difference of the two means $\mu_{1}$ and $\mu_{2}$ is given as

$$
\theta \mid x \sim \bar{x}_{1}-\bar{x}_{2}-\left(T_{1} \sqrt{\frac{\left(n_{1}-1\right) S_{1}^{2}+2 \beta_{1}}{n_{1}\left(n_{1}+2 \alpha_{1}-5\right)}}-T_{2} \sqrt{\frac{\left(n_{2}-1\right) S_{2}^{2}+2 \beta_{2}}{n_{2}\left(n_{2}+2 \alpha_{2}-5\right)}}\right)
$$

and

$$
E(\theta \mid x)=\bar{x}_{1}-\bar{x}_{2}
$$

where $T_{i}$ is a random variable that follows a $t$ distribution with $n_{i}+2 \alpha_{i}-5$ degrees of freedom, where $i=1,2$. The Bayesian measure of evidence under a Gamma Prior is then given by

$$
P_{G a}^{B F}(x)=P\left(\left|T_{1} \sqrt{\frac{\left(n_{1}-1\right) S_{1}^{2}+2 \beta_{1}}{n_{1}\left(n_{1}+2 \alpha_{1}-5\right)}}-T_{2} \sqrt{\frac{\left(n_{2}-1\right) S_{2}^{2}+2 \beta_{2}}{n_{2}\left(n_{2}+2 \alpha_{2}-5\right)}}\right| \geq\left|\bar{x}_{1}-\bar{x}_{2}\right|\right)
$$

To establish that the Bayesian measure of evidence of Yin (2012) solves the paradox in Lindley (1957) when a Gamma prior is assigned to the nuisance parameters, we need to show that

$$
\lim _{\left(n_{1}, n_{2}\right) \rightarrow(\infty, \infty)} P_{G a}^{B F}(x)=0
$$

Recall that

$$
\begin{aligned}
P^{B}(x) & =P\left(|\theta-E(\theta \mid x)| \geq\left|\theta_{0}-E(\theta \mid x)\right| \mid x\right) \\
& =2\left[1-P\left(Z<Z_{0}\right)\right] \\
& =2\left[1-P\left(\chi_{1}^{2}<Z_{0}^{2}\right)\right]
\end{aligned}
$$


where $Z$ is a standard normal random variable. Now, under the Gamma prior, we have that

$$
\begin{aligned}
& Z_{0}^{2}=\frac{\theta_{0}-E(\theta \mid x)}{\sqrt{\operatorname{Var}(\theta \mid x)}} \\
& Z_{0}^{2}=\frac{n_{1}\left(n_{1}+2 \alpha_{1}-7\right) n_{2}\left(n_{2}+2 \alpha_{2}-7\right)\left[\theta_{0}-\left(\bar{x}_{1}-\bar{x}_{2}\right)\right]^{2}}{n_{2}\left(n_{2}+2 \alpha_{2}-7\right)\left[\left(n_{1}-1\right) S_{1}^{2}+2 \beta_{1}\right]+n_{1}\left(n_{1}+2 \alpha_{1}-7\right)\left[\left(n_{2}-1\right) S_{2}^{2}+2 \beta_{2}\right]}
\end{aligned}
$$

Then, it can easily be shown that

$$
\lim _{\left(n_{1}, n_{2}\right) \rightarrow(\infty, \infty)} Z_{0}^{2}=\infty
$$

which implies that (15) holds. To show that (17) holds, we now need to show that

$$
\lim _{n_{1} \rightarrow \infty}\left[\lim _{n_{2} \rightarrow \infty} Z_{0}^{2}\right]=\lim _{n_{2} \rightarrow \infty}\left[\lim _{n_{1} \rightarrow \infty} Z_{0}^{2}\right]=\infty
$$

Let $f_{1}=\lim _{n_{1} \rightarrow \infty}\left[\lim _{n_{2} \rightarrow \infty} Z_{0}^{2}\right], a_{1}=2 \alpha_{1}-7, a_{2}=2 \alpha_{2}-7$ and $A=\left[\theta_{0}-\left(\bar{x}_{1}-\bar{x}_{2}\right)\right]^{2}$ then we have that

$$
\begin{aligned}
& \lim _{n_{2} \rightarrow \infty} Z_{0}^{2}=\lim _{n_{2} \rightarrow \infty}\left[\frac{\left(n_{1}^{2}+n_{1} a_{1}\right)\left(n_{2}^{2}+n_{2} a_{2}\right) A}{\left(n_{2}^{2}+n_{2} a_{2}\right)\left[\left(n_{1}-1\right) S_{1}^{2}+2 \beta_{1}\right]+\left(n_{1}^{2}+n_{1} a_{1}\right)\left[\left(n_{2}-1\right) S_{2}^{2}+2 \beta_{2}\right]}\right] \\
& =\lim _{n_{2} \rightarrow \infty}\left[\frac{n_{2}^{2}\left(n_{1}^{2}+n_{1} a_{1}\right)\left(1+\frac{a_{2}}{n_{2}}\right) A}{n_{2}^{2}\left(1+\frac{a_{2}}{n_{2}}\right)\left[\left(n_{1}-1\right) S_{1}^{2}+2 \beta_{1}\right]+n_{2}\left(n_{1}^{2}+n_{1} a_{1}\right)\left[\left(1-\frac{1}{n_{2}}\right) S_{2}^{2}+\frac{2 \beta_{2}}{n_{2}}\right]}\right] \\
& =\lim _{n_{2} \rightarrow \infty}\left[\frac{\left(n_{1}^{2}+n_{1} a_{1}\right)\left(1+\frac{a_{2}}{n_{2}}\right) A}{\left(1+\frac{a_{2}}{n_{2}}\right)\left[\left(n_{1}-1\right) S_{1}^{2}+2 \beta_{1}\right]+n_{2}^{-1}\left(n_{1}^{2}+n_{1} a_{1}\right)\left[\left(1-\frac{1}{n_{2}}\right) S_{2}^{2}+\frac{2 \beta_{2}}{n_{2}}\right]}\right] \\
& =\frac{\left(n_{1}^{2}+n_{1} a_{1}\right) A}{\left(n_{1}-1\right) S_{1}^{2}+2 \beta_{1}}\left[\frac{\left(n_{1}+a_{1}\right) A}{\left.\left(1-\frac{1}{n_{1}}\right) S_{1}^{2}+\frac{2 \beta_{1}}{n_{1}}\right]}\right]=\infty
\end{aligned}
$$

Secondly, let $f_{2}=\lim _{n_{2} \rightarrow \infty}\left[\lim _{n_{1} \rightarrow \infty} Z_{0}^{2}\right]$, then we have that

$$
\begin{aligned}
& \lim _{n_{1} \rightarrow \infty} Z_{0}^{2}=\lim _{n_{1} \rightarrow \infty}\left[\frac{\left(n_{1}^{2}+n_{1} a_{1}\right)\left(n_{2}^{2}+n_{2} a_{2}\right) A}{\left(n_{2}^{2}+n_{2} a_{2}\right)\left[\left(n_{1}-1\right) S_{1}^{2}+2 \beta_{1}\right]+\left(n_{1}^{2}+n_{1} a_{1}\right)\left[\left(n_{2}-1\right) S_{2}^{2}+2 \beta_{2}\right]}\right] \\
& =\lim _{n_{1} \rightarrow \infty}\left[\frac{n_{1}^{2}\left(1+\frac{a_{1}}{n_{1}}\right)\left(n_{2}^{2}+n_{2} a_{2}\right) A}{\left(n_{2}^{2}+n_{2} a_{2}\right) n_{1}\left[\left(1-\frac{1}{n_{1}}\right) S_{1}^{2}+\frac{2 \beta_{1}}{n_{1}}\right]+n_{1}^{2}\left(1+\frac{a_{1}}{n_{1}}\right)\left[\left(n_{2}-1\right) S_{2}^{2}+2 \beta_{2}\right]}\right]
\end{aligned}
$$




$$
\begin{gathered}
=\lim _{n_{1} \rightarrow \infty}\left[\frac{\left(1+\frac{a_{1}}{n_{1}}\right)\left(n_{2}^{2}+n_{2} a_{2}\right) A}{n_{1}^{-1}\left(n_{2}^{2}+n_{2} a_{2}\right)\left[\left(1-\frac{1}{n_{1}}\right) S_{1}^{2}+\frac{2 \beta_{1}}{n_{1}}\right]+\left(1+\frac{a_{1}}{n_{1}}\right)\left[\left(n_{2}-1\right) S_{2}^{2}+2 \beta_{2}\right]}\right] \\
\lim _{n_{1} \rightarrow \infty} Z_{0}^{2}=\frac{\left(n_{2}^{2}+n_{2} a_{2}\right) A}{\left(n_{2}-1\right) S_{2}^{2}+2 \beta_{2}} \\
f_{2}=\lim _{n_{2} \rightarrow \infty}\left[\frac{\left(n_{2}^{2}+n_{2} a_{2}\right) A}{\left(n_{2}-1\right) S_{2}^{2}+2 \beta_{2}}\right]=\lim _{n_{2} \rightarrow \infty}\left[\frac{\left(n_{2}+a_{2}\right) A}{\left(1-\frac{1}{n_{2}}\right) S_{2}^{2}+\frac{2 \beta_{2}}{n_{2}}}\right]=\infty
\end{gathered}
$$

Since from (19) and (20) we have that $f_{1}=f_{2}=\infty$, it has been shown that the Bayesian measure of evidence of Yin (2012) under the Gamma prior solves the paradox in Lindley[3].

Consequently, since it can be easily seen from (13) that the posterior distribution of $\theta$ is symmetric about its expected value, $E(\theta \mid x)=\bar{x}_{1}-\bar{x}_{2}$, then Theorem 2 of Yin and Li [2] applies here. This implies that under the Gamma Prior, the Bayesian measure of evidence of Yin [1] yields the $1-\alpha$ credible intervals for $\theta=\mu_{1}-\mu_{2}$ centered at $\bar{x}_{1}-\bar{x}_{2}$.

Lemma 1. Let Gamma Priors be assigned to the precisions $\tau_{1}$ and $\tau_{2}$. Then, for values of $\beta_{1} \ll 1, \beta_{2} \ll 1$ and $\alpha_{1}=\alpha_{2}=2$, the Posterior distribution of $\theta$, denoted by $\theta \mid x$ is the same as the Posterior distribution under Jeffreys' independent prior given by $\pi\left(\mu_{1}, \mu_{2}, \sigma_{1}^{2}, \sigma_{2}^{2}\right) \propto \sigma_{1}^{-2} \sigma_{2}^{-2}$

Proof. By considering the values of $\beta_{1}$ and $\beta_{2}$ that satisfy $\beta_{1} \ll 1$ and $\beta_{2} \ll 1$, we can safely assume that

$$
\frac{\beta_{1}}{\left(n_{1}-1\right) S_{1}^{2}} \approx 0 \text { and } \frac{\beta_{2}}{\left(n_{2}-1\right) S_{2}^{2}} \approx 0
$$

especially where $S_{i}^{2} \gg 1$, and $n_{i}$ is sufficiently large, $\forall i=1,2$. Then by setting $\alpha_{1}=\alpha_{2}=2$,we have from (10) that

$$
\begin{aligned}
\pi^{G a}\left(\mu_{1}, \mu_{2} \mid x\right) & \propto\left[1+\frac{n_{1}\left(\bar{x}_{1}-\mu_{1}\right)^{2}}{\left(n_{1}-1\right) S_{1}^{2}}\right]^{-n_{1} / 2}\left[1+\frac{n_{2}\left(\bar{x}_{2}-\mu_{2}\right)^{2}}{\left(n_{2}-1\right) S_{2}^{2}}\right]^{-n_{2} / 2} \\
& \propto\left[1+\frac{t_{1}^{2}}{n_{1}-1}\right]^{-n_{1} / 2}\left[1+\frac{t_{2}^{2}}{n_{2}-1}\right]^{-n_{2} / 2}
\end{aligned}
$$

which is the kernel of the joint distribution of two independent $t$ random variables with $t_{1}$ having $n_{1}-1$ degrees of freedom and $t_{2}$ having $n_{2}-1$ degrees of freedom respectively. The nit can be easily seen that

$$
\mu_{i}=\bar{x}_{i}-\frac{t_{i} S_{i}}{\sqrt{n_{i}}}, i=1,2 .
$$

and consequently, 


$$
\theta \mid x \sim \bar{x}_{1}-\bar{x}_{2}-\left(\frac{S_{1} T_{1}}{\sqrt{n_{1}}}-\frac{S_{2} T_{2}}{\sqrt{n_{2}}}\right)
$$

where $T_{i}$ is a $t$ random variable with $n_{i}-1$ degrees of freedom.

Lemma 1 shows that the posterior distribution of the difference in means under Jeffreys' independent prior is a special case of the posterior distribution of the difference in means under the Gamma prior.

\section{Simulation Results and Discussion}

For the purpose of this discussion, we shall refer to the methodology of Yin [1] as the New Bayesian measure of evidence. The Metropolis-Hastings algorithm was used for the simulation with a thinning length of 12 . The values in Table 1 were obtained by fixing the following values:

$\alpha_{1}=1.5, \alpha_{2}=2.0, \beta_{1}=0.5, \beta_{2}=0.5, S_{1}^{2}=11, S_{2}^{2}=14$. These results reveal that for the different sample sizes, whether large or small, equal or unequal, the conclusions of a hypothesis test based on either the Generalized p-value, the Posterior Predictive p-value, the New Bayesian measure of evidence under the objective prior, or the New Bayesian measure of evidence under the Gamma prior are in the same direction. However, the New Bayesian measure of evidence under the Gamma prior gives consistently smaller evidence against the null hypothesis, whether the sample sizes are equal or unequal except for large sample sizes where the new Bayesian measure of evidence gives stronger evidence against the null compared to the Posterior Predictive P-value.

On the other hand, the values in Table 2 were obtained by fixing the following values: $\alpha_{1}=2.0, \alpha_{2}=2.0, S_{1}^{2}=20, S_{2}^{2}=25$. The values in this table reflect the accuracy of the approximation of the new Bayesian measure of evidence under Jeffreys' independent prior to the new Bayesian measure of evidence under the Gamma prior. Results here show that when the sample sizes are at least 30, the approximation seems to be good and the values of the $\beta_{i}^{\prime} s$ do not need to be far less than 1 . The approximation is good only contingent on the fact that the values of the $\beta_{i}^{\prime} s$ are less than 1.

Thirdly, the values in Table 3 were obtained by fixing the following values: $\alpha_{1}=2.0, \alpha_{2}=2.0, S_{1}^{2}=3, S_{2}^{2}=5$. The values in this table also reflect for smaller variances, the accuracy of the approximation of the new Bayesian measure of evidence under Jeffreys' independent prior by the new Bayesian measure of evidence under the Gamma prior. In a similar manner, results here show that the approximation is equally good for smaller variances. In fact, the approximation is good where samples sizes can be at least as large as 10 so long as the values of the $\beta_{i}^{\prime} s$ are considerably less than 1 . Note that the parameter values are fixed to demonstrate the behaviour of the conclusion from the New Bayesian measure of evidence under different circumstances like when the sample variances are small or moderate or large. Also, in Table 2, the values were fixed to see how well the New Bayesian measure of evidence under Jeffreys' prior can be approximated by the New Bayesian measure of evidence under the Gamma prior. 
Table 1. The four different probability values for different values of $n_{1}$ and $n_{2}$.

\begin{tabular}{|c|c|c|c|c|c|c|c|c|c|}
\hline \multicolumn{10}{|c|}{$n_{1}=3, n_{2}=3$} \\
\hline$\left(\bar{x}_{1}-\bar{x}_{2}\right)$ & 2.500 & 2.200 & 1.900 & 1.600 & 1.300 & 1.000 & 0.700 & 0.400 & 0.100 \\
\hline$p(x)$ & 0.4353 & 0.4885 & 0.5464 & 0.6089 & 0.6758 & 0.7465 & 0.8203 & 0.8965 & 0.9740 \\
\hline $\operatorname{ppp}(x)$ & 0.3685 & 0.4170 & 0.4732 & 0.5375 & 0.6100 & 0.6905 & 0.7781 & 0.8711 & 0.9675 \\
\hline$P_{J}^{B F}$ & 0.5412 & 0.5877 & 0.6363 & 0.6880 & 0.7425 & 0.7993 & 0.8584 & 0.9186 & 0.9795 \\
\hline$P_{G a}^{B F}$ & 0.6829 & 0.7165 & 0.7515 & 0.7878 & 0.8259 & 0.8653 & 0.9050 & 0.9454 & 0.9864 \\
\hline \multicolumn{10}{|c|}{$n_{1}=10, n_{2}=10$} \\
\hline$\left(\bar{x}_{1}-\bar{x}_{2}\right)$ & 2.500 & 2.200 & 1.900 & 1.600 & 1.300 & 1.000 & 0.700 & 0.400 & 0.100 \\
\hline$p(x)$ & 0.1313 & 0.1811 & 0.2451 & 0.3250 & 0.4217 & 0.5350 & 0.6632 & 0.8031 & 0.9503 \\
\hline $\operatorname{ppp}(x)$ & 0.1344 & 0.1790 & 0.2374 & 0.3120 & 0.4051 & 0.5172 & 0.6476 & 0.7928 & 0.9475 \\
\hline$P_{J}^{B F}$ & 0.1546 & 0.2069 & 0.2723 & 0.3524 & 0.4474 & 0.5574 & 0.6801 & 0.8131 & 0.9531 \\
\hline$P_{G a}^{B F}$ & 0.1692 & 0.2229 & 0.2896 & 0.3694 & 0.4634 & 0.5718 & 0.6911 & 0.8203 & 0.9548 \\
\hline \multicolumn{10}{|c|}{$n_{1}=30, n_{2}=30$} \\
\hline$\left(\bar{x}_{1}-\bar{x}_{2}\right)$ & 2.500 & 2.200 & 1.900 & 1.600 & 1.300 & 1.000 & 0.700 & 0.400 & 0.100 \\
\hline$p(x)$ & 0.0082 & 0.0191 & 0.0418 & 0.0849 & 0.1598 & 0.2778 & 0.4463 & 0.6629 & 0.9131 \\
\hline $\operatorname{ppp}(x)$ & 0.0112 & 0.0229 & 0.0457 & 0.0876 & 0.1598 & 0.2746 & 0.4408 & 0.6580 & 0.9117 \\
\hline$P_{J}^{B F}$ & 0.0094 & 0.0214 & 0.0456 & 0.0909 & 0.1675 & 0.2856 & 0.4531 & 0.6675 & 0.9144 \\
\hline$P_{G a}^{B F}$ & 0.0102 & 0.0229 & 0.0480 & 0.0939 & 0.1714 & 0.2909 & 0.4580 & 0.6709 & 0.9154 \\
\hline \multicolumn{10}{|c|}{$n_{1}=3, n_{2}=5$} \\
\hline$\left(\bar{x}_{1}-\bar{x}_{2}\right)$ & 2.500 & 2.200 & 1.900 & 1.600 & 1.300 & 1.000 & 0.700 & 0.400 & 0.100 \\
\hline$p(x)$ & 0.3635 & 0.4202 & 0.4832 & 0.5524 & 0.6275 & 0.7077 & 0.7923 & 0.8802 & 0.9699 \\
\hline$p p p(x)$ & 0.3212 & 0.3708 & 0.4292 & 0.4969 & 0.5743 & 0.6612 & 0.7564 & 0.8583 & 0.9643 \\
\hline$P_{J}^{B F}$ & 0.4579 & 0.5094 & 0.5654 & 0.6255 & 0.6898 & 0.7576 & 0.8284 & 0.9010 & 0.9754 \\
\hline$P_{G a}^{B F}$ & 0.6340 & 0.6712 & 0.7108 & 0.7525 & 0.7960 & 0.8414 & 0.8883 & 0.9359 & 0.9838 \\
\hline \multicolumn{10}{|c|}{$n_{1}=12, n_{2}=15$} \\
\hline$\left(\bar{x}_{1}-\bar{x}_{2}\right)$ & 2.500 & 2.200 & 1.900 & 1.600 & 1.300 & 1.000 & 0.700 & 0.400 & 0.100 \\
\hline$p(x)$ & 0.0780 & 0.1183 & 0.1747 & 0.2505 & 0.3483 & 0.4691 & 0.6113 & 0.7711 & 0.9420 \\
\hline $\operatorname{ppp}(x)$ & 0.0843 & 0.1214 & 0.1734 & 0.2443 & 0.3378 & 0.4561 & 0.5989 & 0.7625 & 0.9396 \\
\hline$P_{J}^{B F}$ & 0.0917 & 0.1348 & 0.1936 & 0.2712 & 0.3686 & 0.4869 & 0.6258 & 0.7798 & 0.9441 \\
\hline$P_{G a}^{B F}$ & 0.1020 & 0.1468 & 0.2073 & 0.2852 & 0.3824 & 0.4997 & 0.6359 & 0.7862 & 0.9461 \\
\hline \multicolumn{10}{|c|}{$n_{1}=40, n_{2}=35$} \\
\hline$\left(\bar{x}_{1}-\bar{x}_{2}\right)$ & 2.500 & 2.200 & 1.900 & 1.600 & 1.300 & 1.000 & 0.700 & 0.400 & 0.100 \\
\hline$p(x)$ & 0.0033 & 0.0091 & 0.0236 & 0.0553 & 0.1179 & 0.2275 & 0.3970 & 0.6278 & 0.9035 \\
\hline $\operatorname{ppp}(x)$ & 0.0050 & 0.0117 & 0.0268 & 0.0584 & 0.1193 & 0.2260 & 0.3929 & 0.6236 & 0.9021 \\
\hline
\end{tabular}




\section{Continued}

\begin{tabular}{cccccccccc}
\hline$P_{J}^{B F}$ & 0.0038 & 0.0105 & 0.0260 & 0.0592 & 0.1233 & 0.2343 & 0.4039 & 0.6327 & 0.9044 \\
$P_{G a}^{B F}$ & 0.0040 & 0.0110 & 0.0269 & 0.0607 & 0.1257 & 0.2371 & 0.4065 & 0.6348 & 0.9052 \\
\hline & & & & $n_{1}=100, n_{2}=110$ & & & & \\
\hline$\left(\bar{x}_{1}-\bar{x}_{2}\right)$ & 2.500 & 2.200 & 1.900 & 1.600 & 1.300 & 1.000 & 0.700 & 0.400 & 0.100 \\
$p(x)$ & 0.0000 & 0.0000 & $1 \mathrm{e}-04$ & 0.0012 & 0.0082 & 0.0413 & 0.1522 & 0.4125 & 0.8375 \\
$p p p(x)$ & 0.0000 & 0.0000 & $2 \mathrm{e}-04$ & 0.0015 & 0.0090 & 0.0424 & 0.1523 & 0.4110 & 0.8368 \\
$P_{J}^{B F}$ & 0.0000 & 0.0000 & $1 \mathrm{e}-04$ & 0.0013 & 0.0087 & 0.0426 & 0.1548 & 0.4157 & 0.8386 \\
$P_{G a}^{B F}$ & 0.0000 & 0.0000 & $1 \mathrm{e}-04$ & 0.0013 & 0.0088 & 0.0432 & 0.1546 & 0.4149 & 0.8388 \\
\hline
\end{tabular}

Table 2. The four different probability values for different values of $\alpha_{1}, \alpha_{2}, \beta_{1}$ and $\beta_{2}$.

\begin{tabular}{|c|c|c|c|c|c|c|c|c|c|}
\hline \multicolumn{10}{|c|}{$\alpha_{1}=0.5, \alpha_{2}=0.5, \beta_{1}=2.5, \beta_{2}=2.5$} \\
\hline$\left(\bar{x}_{1}-\bar{x}_{2}\right)$ & 2.500 & 2.200 & 1.900 & 1.600 & 1.300 & 1.000 & 0.700 & 0.400 & 0.100 \\
\hline$p(x)$ & 0.0022 & 0.0068 & 0.0186 & 0.0464 & 0.1041 & 0.2097 & 0.3786 & 0.6143 & 0.8997 \\
\hline$p p p(x)$ & 0.0035 & 0.0088 & 0.0214 & 0.0492 & 0.1056 & 0.2085 & 0.3750 & 0.6104 & 0.8984 \\
\hline$P_{J}^{B F}$ & 0.0025 & 0.0073 & 0.0202 & 0.0493 & 0.1086 & 0.2151 & 0.3840 & 0.6182 & 0.9006 \\
\hline$P_{G a}^{B F}$ & 0.0040 & 0.0107 & 0.0267 & 0.0612 & 0.1263 & 0.2373 & 0.4077 & 0.6347 & 0.9053 \\
\hline \multicolumn{10}{|c|}{$\alpha_{1}=0.5, \alpha_{2}=1.5, \beta_{1}=2.5, \beta_{2}=2.0$} \\
\hline$\left(\bar{x}_{1}-\bar{x}_{2}\right)$ & 2.500 & 2.200 & 1.900 & 1.600 & 1.300 & 1.000 & 0.700 & 0.400 & 0.100 \\
\hline$p(x)$ & 0.0022 & 0.0068 & 0.0186 & 0.0464 & 0.1041 & 0.2097 & 0.3786 & 0.6143 & 0.8997 \\
\hline $\operatorname{ppp}(x)$ & 0.0035 & 0.0088 & 0.0214 & 0.0492 & 0.1056 & 0.2085 & 0.3750 & 0.6104 & 0.8984 \\
\hline$P_{J}^{B F}$ & 0.0025 & 0.0073 & 0.0202 & 0.0493 & 0.1086 & 0.2151 & 0.3840 & 0.6182 & 0.9006 \\
\hline$P_{G a}^{B F}$ & 0.0034 & 0.0094 & 0.0242 & 0.0568 & 0.1202 & 0.2301 & 0.3998 & 0.6297 & 0.9040 \\
\hline \multicolumn{10}{|c|}{$\alpha_{1}=2, \alpha_{2}=2, \beta_{1}=0.0005, \beta_{2}=0.0005$} \\
\hline$\left(\bar{x}_{1}-\bar{x}_{2}\right)$ & 2.500 & 2.200 & 1.900 & 1.600 & 1.300 & 1.000 & 0.700 & 0.400 & 0.100 \\
\hline$p(x)$ & 0.0022 & 0.0068 & 0.0186 & 0.0464 & 0.1041 & 0.2097 & 0.3786 & 0.6143 & 0.8997 \\
\hline $\operatorname{ppp}(x)$ & 0.0035 & 0.0088 & 0.0214 & 0.0492 & 0.1056 & 0.2085 & 0.3750 & 0.6104 & 0.8984 \\
\hline$P_{J}^{B F}$ & 0.0025 & 0.0073 & 0.0202 & 0.0493 & 0.1086 & 0.2151 & 0.3840 & 0.6182 & 0.9006 \\
\hline$P_{G a}^{B F}$ & 0.0026 & 0.0076 & 0.0202 & 0.0494 & 0.1088 & 0.2154 & 0.3852 & 0.6191 & 0.9007 \\
\hline \multicolumn{10}{|c|}{$\alpha_{1}=4, \alpha_{2}=5, \beta_{1}=2.5, \beta_{2}=2.0$} \\
\hline$\left(\bar{x}_{1}-\bar{x}_{2}\right)$ & 2.500 & 2.200 & 1.900 & 1.600 & 1.300 & 1.000 & 0.700 & 0.400 & 0.100 \\
\hline$p(x)$ & 0.0022 & 0.0068 & 0.0186 & 0.0464 & 0.1041 & 0.2097 & 0.3786 & 0.6143 & 0.8997 \\
\hline $\operatorname{ppp}(x)$ & 0.0035 & 0.0088 & 0.0214 & 0.0492 & 0.1056 & 0.2085 & 0.3750 & 0.6104 & 0.8984 \\
\hline$P_{J}^{B F}$ & 0.0025 & 0.0073 & 0.0202 & 0.0493 & 0.1086 & 0.2151 & 0.3840 & 0.6182 & 0.9006 \\
\hline$P_{G a}^{B F}$ & 0.0014 & 0.0044 & 0.0134 & 0.0361 & 0.0882 & 0.1877 & 0.3551 & 0.5960 & 0.8945 \\
\hline
\end{tabular}


Table 3. The four different probability values for different values of $\alpha_{1}, \alpha_{2}, \beta_{1}$ and $\beta_{2}$.

\begin{tabular}{|c|c|c|c|c|c|c|c|c|c|}
\hline \multicolumn{10}{|c|}{$\alpha_{1}=2, \alpha_{2}=2, \beta_{1}=0.0005, \beta_{2}=0.0005$} \\
\hline$\left(\bar{x}_{1}-\bar{x}_{2}\right)$ & 2.500 & 2.200 & 1.900 & 1.600 & 1.300 & 1.000 & 0.700 & 0.400 & 0.100 \\
\hline$p(x)$ & 0.0000 & 0.0000 & 0.0000 & 0.0000 & 0.0001 & 0.0026 & 0.0329 & 0.2189 & 0.7577 \\
\hline$p p p(x)$ & 0.0000 & 0.0000 & 0.0000 & 0.0000 & 0.0003 & 0.0037 & 0.0353 & 0.2178 & 0.7553 \\
\hline$P_{J}^{B F}$ & 0.0000 & 0.0000 & 0.0000 & 0.0000 & 0.0002 & 0.0028 & 0.0349 & 0.2244 & 0.7611 \\
\hline$P_{G a}^{B F}$ & 0.0000 & 0.0000 & 0.0000 & 0.0000 & 0.0001 & 0.0028 & 0.0349 & 0.2234 & 0.7592 \\
\hline \multicolumn{10}{|c|}{$\alpha_{1}=2, \alpha_{2}=1.2, \beta_{1}=3.5, \beta_{2}=3.0$} \\
\hline$\left(\bar{x}_{1}-\bar{x}_{2}\right)$ & 2.500 & 2.200 & 1.900 & 1.600 & 1.300 & 1.000 & 0.700 & 0.400 & 0.100 \\
\hline$p(x)$ & 0.0000 & 0.0000 & 0.0000 & 0.0000 & 0.0001 & 0.0026 & 0.0329 & 0.2189 & 0.7577 \\
\hline $\operatorname{ppp}(x)$ & 0.0000 & 0.0000 & 0.0000 & 0.0000 & 0.0003 & 0.0037 & 0.0353 & 0.2178 & 0.7553 \\
\hline$P_{J}^{B F}$ & 0.0000 & 0.0000 & 0.0000 & 0.0000 & 0.0002 & 0.0028 & 0.0349 & 0.2244 & 0.7611 \\
\hline$P_{G a}^{B F}$ & 0.0000 & 0.0000 & 0.0000 & 0.0000 & 0.0002 & 0.0041 & 0.0418 & 0.2402 & 0.7678 \\
\hline \multicolumn{10}{|c|}{$\alpha_{1}=0.3, \alpha_{2}=0.7, \beta_{1}=15, \beta_{2}=10$} \\
\hline$\left(\bar{x}_{1}-\bar{x}_{2}\right)$ & 2.500 & 2.200 & 1.900 & 1.600 & 1.300 & 1.000 & 0.700 & 0.400 & 0.100 \\
\hline$p(x)$ & 0.0000 & 0.0000 & 0.0000 & 0.0000 & 0.0001 & 0.0026 & 0.0329 & 0.2189 & 0.7577 \\
\hline $\operatorname{ppp}(x)$ & 0.0000 & 0.0000 & 0.0000 & 0.0000 & 0.0003 & 0.0037 & 0.0353 & 0.2178 & 0.7553 \\
\hline$P_{J}^{B F}$ & 0.0000 & 0.0000 & 0.0000 & 0.0000 & 0.0002 & 0.0028 & 0.0349 & 0.2244 & 0.7611 \\
\hline$P_{G a}^{B F}$ & 0.0000 & 0.0000 & 0.0000 & 0.0000 & 0.0008 & 0.0090 & 0.0644 & 0.2871 & 0.7891 \\
\hline \multicolumn{10}{|c|}{$\alpha_{1}=9, \alpha_{2}=4, \beta_{1}=15, \beta_{2}=10$} \\
\hline$\left(\bar{x}_{1}-\bar{x}_{2}\right)$ & 2.500 & 2.200 & 1.900 & 1.600 & 1.300 & 1.000 & 0.700 & 0.400 & 0.100 \\
\hline$p(x)$ & 0.0000 & 0.0000 & 0.0000 & 0.0000 & 0.0001 & 0.0026 & 0.0329 & 0.2189 & 0.7577 \\
\hline $\operatorname{ppp}(x)$ & 0.0000 & 0.0000 & 0.0000 & 0.0000 & 0.0003 & 0.0037 & 0.0353 & 0.2178 & 0.7553 \\
\hline$P_{J}^{B F}$ & 0.0000 & 0.0000 & 0.0000 & 0.0000 & 0.0002 & 0.0028 & 0.0349 & 0.2244 & 0.7611 \\
\hline$P_{G a}^{B F}$ & 0.0000 & 0.0000 & 0.0000 & 0.0000 & 0.0002 & 0.0032 & 0.0369 & 0.2299 & 0.7631 \\
\hline
\end{tabular}

Finally, Lehmann's data on measures of driving times from following two different routes and Sahu's data on scores of surgical and non-surgical treatments both displayed as Table 1 and Table 2 respectively in Yin and Li [2] were used as real examples to demonstrate the performance of the four measures of evidence (results not shown). All conclusions were in the same direction for all four measures of evidence against the null hypothesis.

\section{Conclusions}

In this paper, we looked at the Bayesian analysis of the Behrens-Fisher problem using the methodology of Yin [1] by assigning Gamma Priors to the two un- 
known variances. We were able to show analytically, that the Bayesian measure of evidence of Yin [1] solves simultaneously, the Behrens-Fisher problem and Lindley's paradox when Gamma Priors are assigned to the unknown variances. In fact, we were able to show that the solution obtained by Yin and Li [2] is a special case of ours, where Gamma Prior is used instead of Jeffreys' independent prior.

Simulation results further confirm the fact that extending the methodology of Yin [1] while assigning Gamma Prior to each of the nuisance parameters also solves Lindley's paradox. This implies that the prowess of the methodology of Yin [1] does not only lie in the use of noninformative priors. In fact, simulation results reveal that for large sample sizes, the measure of evidence against the null hypothesis is stronger when the nuisance parameters are assigned Gamma Priors with carefully selected parameter values.

\section{Conflicts of Interest}

The authors declare no conflicts of interest regarding the publication of this paper.

\section{References}

[1] Yin, Y. (2012) A New Bayesian Procedure for Testing Point Null Hypothesis. Computational Statistics, 27, 237-249. https://doi.org/10.1007/s00180-011-0252-6

[2] Yin, Y. and Li, B. (2014) Analysis of the Behrens-Fisher Problem Based on Bayesian Evidence. Journal of Applied Mathematics, 2014, Article ID: 978691. https://doi.org/10.1155/2014/978691

[3] Lindley, D.V. (1957) A Statistical Paradox. Biometrika, 44, 187-192. https://doi.org/10.1093/biomet/44.1-2.187

[4] Spanos, A. (2013) Who Should Be Afraid of the Jeffreys-Lindley Paradox? Philosophy of Science, 80, 73-93. https://doi.org/10.1086/668875

[5] Robert, C.P. (2014) On the Jeffreys-Lindley's Paradox. Philosophy of Science, 81, 216-232. https://doi.org/10.1086/675729

[6] Scheffe, H. (1944) A Note on the Behrens-Fisher Problem. Annals of Mathematical Statistics, 15, 430-432. https://doi.org/10.1214/aoms/1177731214

[7] Fraser, D.A.S. and Streit, F. (1972) On the Behrens-Fisher Problem. Australian Journal of Statistics, 14, 167-171. https://doi.org/10.1111/j.1467-842X.1972.tb00354.x

[8] Robinson, G.K. (1976) Properties of Students $t$ and of the Behrens-Fisher Solution to the Two Means Problem. The Annals of Statistics, 4, 963-971. https://doi.org/10.1214/aos/1176343594

[9] Tsui, K.-W. and Weerahandi, S. (1989) Generalized p-Values in Significance Testing of Hypotheses in the Presence of Nuisance Parameters. Journal of American Statistical Association, 84, 602-607. https://doi.org/10.2307/2289949

[10] Zheng, S., Shi, N.-Z. and Ma, W. (2009) Statistical Inference on the Difference or Ratio of Means from Heteroscedastic Normal Populations. Journal of Statistical Planning and Inference, 140, 1236-1242. https://doi.org/10.1016/j.jspi.2009.11.010

[11] Ozkip, E., Yazici, B. and Sezer, A. (2014) A Simulation Study on Tests for the Be- 
hrens-Fisher Problem. Turkiye Klinikleri Journal of Biostatistics, 6, 59-66.

[12] Degroot, M.H. (1982) Comment. Journal of the American Statistical Association, 77, 336-339. https://doi.org/10.1080/01621459.1982.10477811

[13] Berger, J.O. and Sellke, T. (1987) Testing a Point null Hypothesis: The Irreconcilability of p-Values and Evidence. Journal of the American Statistical Association, 82, 112-122. https://doi.org/10.2307/2289131

[14] Casella, G. and Berger, R.L. (1987) Reconciling Bayesian and Frequentist Evidence in One Sided Testing Problem. Journal of the American Statistical Association, 82, 106-111. https://doi.org/10.1080/01621459.1987.10478396

[15] Berger, J.O. and Delampady, M. (1987) Testing Precise Hypotheses. Statistical Science, 2, 317-352. https://doi.org/10.1214/ss/1177013238

[16] Meng, X.-L. (1994) Posterior Predictive p-Values. The Annals of Statistics, 22, 1142-1160. https://doi.org/10.1214/aos/1176325622

[17] Ghosh, M. and Kim, Y.-H. (2001) The Behrens-Fisher Problem Revisited: A Bayes-Frequentist Synthesis. The Canadian Journal of Statistics, 29, 5-17. https://doi.org/10.2307/3316047 